\title{
Investigation on spectral loss characteristics of subwavelength terahertz fibers
}

\author{
Hung-Wen Chen \\ Department of Electrical Engineering and Graduate Institute of Electro-Optical Engineering, \\ National Taiwan University, Taipei, 10617, Taiwan \\ Yu-Tai Li and Ci-Ling Pan \\ Department of Photonics and Institute of Electro-Optical Engineering, National Chiao Tung University, \\ Hsinchu, 30010, Taiwan \\ Jeng-Liang Kuo \\ Material and Chemical Research Laboratories, Industrial Technology Research Institute, Hsinchu, 30010, Taiwan \\ Ja-Yu Lu, Li-Jin Chen, and Chi-Kuang Sun \\ Department of Electrical Engineering and Graduate Institute of Electro-Optical Engineering, National Taiwan \\ University and Research Center for Applied Sciences, Academia Sinica, Taipei, 10617, Taiwan \\ Received December 1, 2006; revised January 30, 2007; accepted February 1, 2007; \\ posted February 6, 2007 (Doc. ID 77714); published April 3, 2007 \\ By measuring the spectral loss characteristics of subwavelength-diameter terahertz fibers, our study sup- \\ ports the recent theory proposed by M. Sumetsky [Opt. Lett. 31, 870 (2006)] that diameter-variation- \\ induced radiation is a dominant loss mechanism for subwavelength fibers in the low- $(<1 \%)$ core-fraction- \\ power regime. This physical mechanism limits the lowest guidable frequency in a subwavelength fiber. \\ (C) 2007 Optical Society of America \\ OCIS codes: $060.2430,260.3090$.
}

Recently subwavelength optical waveguides and fibers ${ }^{1,2}$ have been successfully demonstrated, not just with a minimized size favorable for photonic device applications, but also with strong light confinement to enhance the device nonlinearity. For terahertz $(\mathrm{THz})$ wave applications, we have also proposed and demonstrated a low-loss subwavelength $\mathrm{THz}$ polyethylene (PE) fiber ${ }^{3}$ for $\mathrm{THz}$ waveguiding, with a structure similar to the optical nanofiber. ${ }^{1}$ However, the purpose for the subwavelength $\mathrm{THz}$ fiber is to reduce the interaction between $\mathrm{THz}$ waves and the fiber core, and the subwavelength $\mathrm{THz}$ fiber is preferred for operation in the low-core-fraction-power region, very different from the optical subwavelength fiber. With a core diameter much smaller than the $\mathrm{THz}$ wavelength, the only sustained $\mathrm{HE}_{11}$ mode allows the majority of the energy to be guided outside the fiber. With a low-core-fraction power, of the order of or less than $1 \%, \mathrm{THz}$ waves can propagate with an attenuation constant value of the order of or less than $1 \%$ of the bulk value, while an effective attenuation constant of $10^{-3} \mathrm{~cm}^{-1}$ was successfully demonstrated. Previous optical nanotaper fiber experiments with a core diameter much smaller than the optical guiding wavelength had found a significant loss increase with reduced fiber radius, ${ }^{1,4}$ which was attributed to possible bending loss. ${ }^{4}$ Based on the theory of nonadiabatic transitions developed in quantum mechanics, M. Sumetsky recently proposed the theory that small nonuniformity of optical subwavelength fiber diameter could induce strong radiation loss when the diameter of the fiber is much smaller than the wavelength. ${ }^{5}$ With a large portion of energy guided outside the subwavelength $\mathrm{THz}$ fiber, this proposed loss mechanism ${ }^{5}$ could thus pose a lower limit on the guidable fraction power in the core. In this Letter we report our investigation on the influence of the nonuniformity-induced radiation loss in a subwavelength $\mathrm{THz}$ fiber in the low-core-fraction-power regime. We studied the spectral loss characteristics of subwavelength $\mathrm{THz}$ fibers and compared the proposed theory ${ }^{5}$ with the experimental results. It is found that diameter-variation-induced radiation loss could be a dominant loss mechanism in subwavelength $\mathrm{THz}$ fibers in the regime of low- $(<1 \%)$ corefractional power.

In this experiment, three different diameter $\mathrm{PE}$ wires were made by a melt-spinning method. We perforated through a $40 \mu \mathrm{m}$ thick PE film attached to an aluminum ring with a needle and created a small aperture slightly smaller than the cross section of the $\mathrm{PE}$ wires (fiber core). We hung the fiber with its two ends each resting in the hole of one PE film oriented transverse to the fiber and fixed each ring on an ordinary 1 in. mirror mount to adjust the tilt of the fiber. Since the hole was smaller than the fiber diameter, the PE films could hold the fiber tight and stable. To investigate the spectral loss characteristics of the subwavelength $\mathrm{THz}$ fiber, we adopted a measurement system composed of a photoconductive (PC) antenna ${ }^{6}$ as a broadband $\mathrm{THz}$ source and a MartinPuplett interferometer ${ }^{7}$ (MPI) as a spectrometer. $\mathrm{THz}$ waves emitted from the PC antenna were collimated into the input of the MPI by an off-axis parabolic mirror; in the output of the MPI they were collected and coupled into the measured subwavelength fiber by 


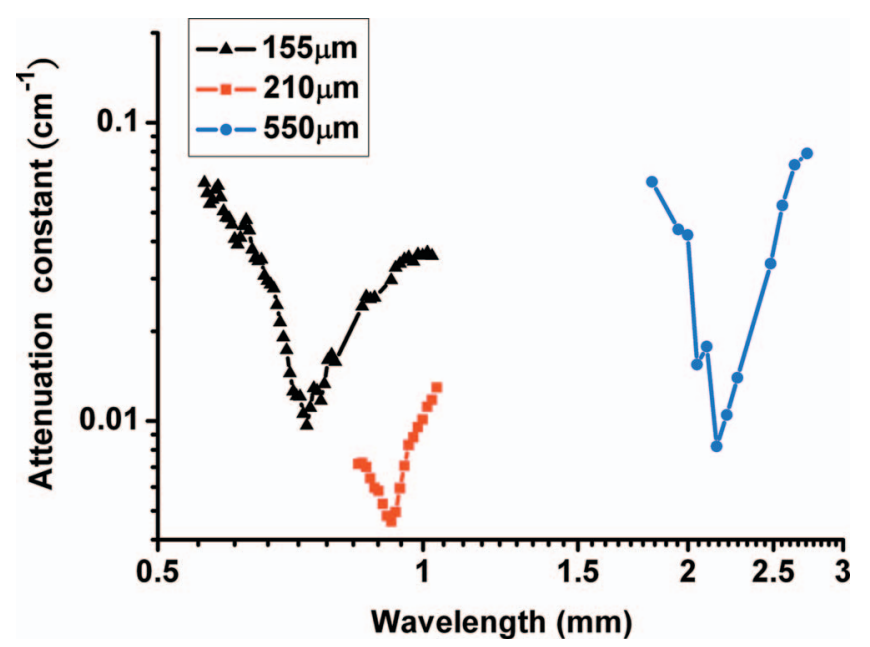

Fig. 1. Propagation loss of three different $\mathrm{THz}$ fibers with diameters of 155,210 , and $550 \mu \mathrm{m}$, as a function of wavelength.

another off-axis parabolic mirror and detected by a $\mathrm{Si}$ bolometer positioned at the output end of the fiber. The spectrum of the fiber output was thus obtained from the measured interferogram.

The longest available lengths for 210, 155, and $550 \mu \mathrm{m}$ diameter fibers are $105,42.5$, and $29 \mathrm{~cm}$, respectively. When performing the experiment, we began with the fiber output measurement with the longest fiber length for different fiber diameters. With the same fiber and the same input coupling, we then cut a small piece of the fiber off from the output end and repeated the measurements. As shown in Fig. 1, the attenuation constants as a function of the wavelength for different fiber diameters can be analyzed from the measured interferogram, after comparing the results from two different fiber lengths. The effect of frequency-dependent coupling loss was thus canceled. Interestingly, we found that melt-spun $\mathrm{PE}$ fibers of different diameters have minimum attenuation constants, typically of the order of $10^{-2}-10^{-3} \mathrm{~cm}^{-1}$ at distinct wavelength ranges, and a thinner fiber delivers $\mathrm{THz}$ waves with minimal attenuation at a shorter wavelength. It should be noted that the length difference between two measured $210 \mu \mathrm{m}$ fibers was much longer than the other two fibers such that the measured interferogram suffered less noise. Therefore the measured attenuation constant of the $210 \mu \mathrm{m}$ fiber can reach a smaller value than those of 155 and $550 \mu \mathrm{m}$ ones. As mentioned in Ref. 3, the dominant loss mechanism for $\mathrm{THz}$ waveguiding is fractional material absorption, which strongly depends on the fractional power inside the waveguide. For a subwavelength fiber, the shorter the guiding wavelength, the larger portion of the power confined in the core, leading to a higher attenuation constant. On the short-wavelength (left) side of the minimum attenuation point, the attenuation constant as expected shows an increasing trend toward smaller wavelength and higher frequency. However, on its long-wavelength (right) side the attenuation constant is increasing as the wavelength increases, in contrast to the expected decreasing trend, owing to the declined fractional power inside the core. This result indicated that fractional material absorption is not the only primary contributor to the loss curve and that there must be another loss mechanism existing in the subwavelength fiber, possibly nonuniformity-induced radiation loss.

The PE fibers in this study were fabricated by a melt-spinning method, which is generally with a coefficient of variation (C.V.) in diameter smaller than $3 \%$. The value of the C.V. is defined as the ratio of the standard deviation to the mean. A subwavelength fiber with a large portion of power outside the core could suffer serious loss from diameter-variationinduced radiation. Slight changes of the core diameter cause the modes of the fiber to couple with one another. If we excite a pure guiding mode at the input end of the fiber, diameter variation could make its mode field couple to the radiation modes, resulting in fiber losses, since the power is carried away from the core into the infinite cladding, as predicted by Sumetsky in optical subwavelength fiber. ${ }^{5}$ In Sumetsky's theory, to provide an analytical solution, some simplification and assumption are adopted, which could result in relatively poor accuracy of the theory. The fiber diameter variation is considered smooth and can be adiabatically shown as a function of the fiber length. The shape of the optical taper fiber is expressed as $d(z)=d_{\infty}-\left(d_{\infty}-d_{0}\right)\{1+[(z$ $\left.\left.\left.-z_{0}\right) / L\right]\right\}^{-1}$, where $d_{0}$ and $d_{\infty}$ are the taper's diameters at its center and ends, respectively. For a silica microtaper with a small waist diameter of $d \ll \lambda$, $\Delta \beta_{12}(z)$, the difference between the propagation constant of the $\mathrm{HE}_{11}$ mode, $\beta_{1}(z)$, and that of the radiation threshold, $\beta_{2}(z)=2 \pi / \lambda$, is approximated as

$$
\Delta \beta_{12}(z) \approx 0.57\left[\lambda / d^{2}(z)\right] \exp \left[-0.27 \lambda^{2} / d^{2}(z)\right] .
$$

Substituting $\Delta \beta_{12}(z)$ into the Landau-Dykhne formula, the fiber transmission loss can thus be roughly estimated.

To apply Sumetsky's theory in our case, the expression of $\Delta \beta_{12}$ is modified according to our numerical

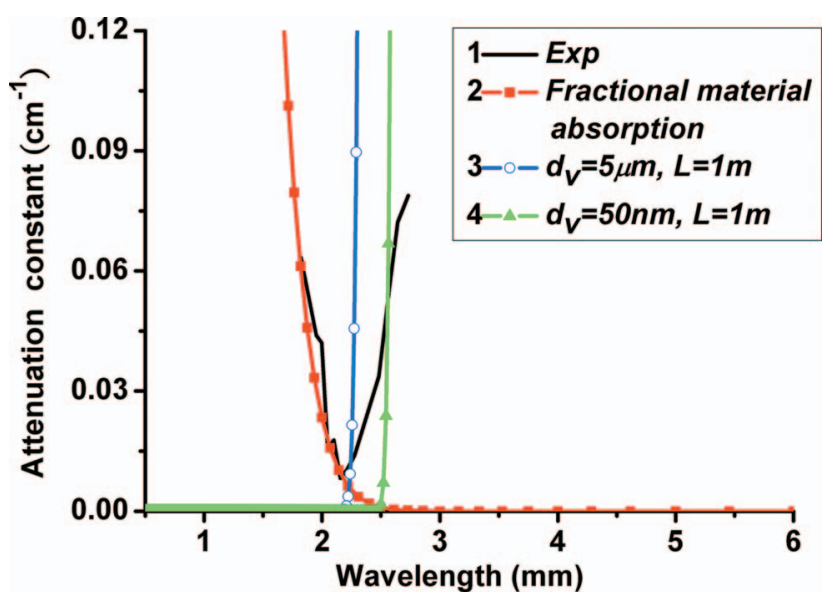

Fig. 2. THz spectral loss characteristic of the $550 \mu \mathrm{m} \mathrm{di-}$ ameter PE fiber. Curve 1, measured result; curve 2, calculated loss due to fractional material absorption; Curves 3 and 4, calculated losses due to diameter-variation-induced radiation with $d_{v}=5 \mu \mathrm{m}$ and $d_{v}=50 \mathrm{~nm}$, respectively. Characteristic length $L$ is assumed to be $1 \mathrm{~m}$. 


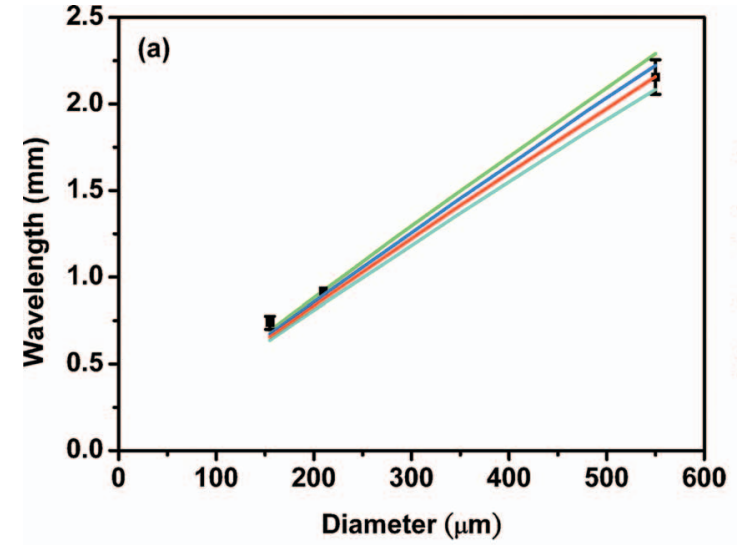

(a)

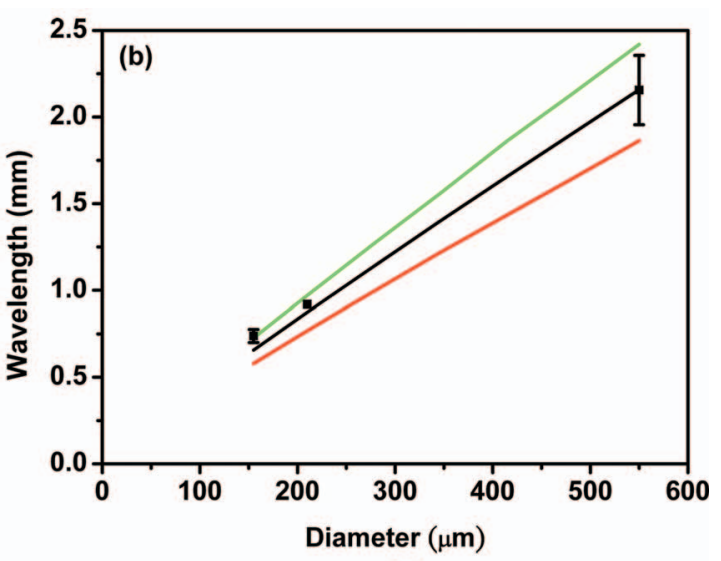

(b)

Fig. 3. (a) Measured minimum attenuation wavelength as a function of fiber diameter. Simulation results with different C.V. values and a fixed $L=1 \mathrm{~m}$ are provided for comparison. Four simulation curves, from top to bottom, correspond to parameters C.V. $=0.3 \%, 1 \%, 3 \%, 10 \%$, respectively. (b) Simulation results with different characteristic lengths and a fixed C.V. value of $3 \%$ are provided for comparison with the experimental results. Three simulation curves, from top to bottom, correspond to parameters $L=10,1,0.1 \mathrm{~m}$, respectively.

results. We combine the calculated loss spectrum from fractional material absorption with that from diameter-variation-induced radiation to compare with our experimental results. The parameters are defined as follows: $d_{0}$ is the fiber diameter, and $d_{\nu}$ $=d_{\infty}-d_{0}$ is the fiber diameter variation. The experimental results and full-modeled results with $d_{\nu}$ $=5 \mu \mathrm{m}$ and $d_{\nu}=50 \mathrm{~nm}$ for a fixed $L=1 \mathrm{~m}$ are shown in Fig. 2. Curve 2 (calculated fractional material absorption) intersects both curves 3 and 4 (calculated diameter-variation-induced radiation loss) at a frequency near the one at which the experimental minimum attenuation constant occurs, indicating that diameter-variation-induced radiation is a dominant loss mechanism in subwavelength $\mathrm{THz}$ fiber when the core-fraction power is low. It should be noted that, just like the experimental curve in Fig. 2 of Ref. 5 , the experimental gradient on the long-wavelength side of the minimum point is much different from those of theoretical curves 3 and 4, owing to the simplified theory. However, one could still successfully utilize Sumetsky's approximation to estimate the wavelength of the minimum attenuation constants. In Fig. 3 we show the measured minimum attenuation wavelength as a function of the PE fiber diameter. The experimental values are also compared with the theoretical value as analyzed in Fig. 3(a) with different coefficients of variation and in Fig. 3(b) with different characteristic lengths. The measured wavelength for the minimum attenuation is about four times the fiber diameter and is close to the numerical estimation with parameters of C.V. $=3 \%$ and $L=1 \mathrm{~m}$. We point out that even with a large C.V. in the studied melt-spun PE fibers, diameter-variation-induced radiation dominates the loss mechanism of the subwavelength fiber only when the fractional power in the core is of the order of or lower than $1 \%$. It should also be noted that even though the $\mathrm{THz}$ source is a broadband PC antenna, the spectra of the fiber attentuation were narrow because of the limited affordable loss after a long PE fiber. Hence the analyzed attenuation constant as a function of the wavelength shown in Fig. 1 was limited in a relatively narrow spectral region with a reasonable signal-to-noise ratio.

In conclusion, we found that there are two dominant loss mechanisms in subwavelength $\mathrm{THz} \mathrm{PE}$ fibers operating in the regime of low fractional power in the core $(<1 \%)$. Combining these two loss mechanisms, fractional material absorption and diametervariation-induced radiation, the predicted minimum attenuation frequency matches the experimental values well. This study also supports the theory proposed by Sumetsky, which provides a physical mechanism that limits the lowest guidable frequency in a subwavelength fiber.

This work was sponsored by the National Science Council of Taiwan under grants 95-2221-E-002-094 and 95-2120-M-002-013, the Program for Promoting Academic Excellence of University Project (Phase II), the National Taiwan University Center for Genomic Medicine, and the Aim for the Top University program of the Ministry of Education, Taiwan. Corresponding author C.-K. Sun's e-mail address is sun@cc.ee.ntu.edu.tw.

\section{References}

1. L. M. Tong, R. R. Gattass, J. B. Ashcom, S. L. He, J. Y. Lou, M. Y. Shen, I. Maxwell, and E. Mazur, Nature 426, 816 (2003).

2. X. F. Duan, Y. Huang, R. Agarwal, and C. M. Lieber, Nature 421, 241 (2003).

3. L. J. Chen, H. W. Chen, T. F. Kao, J. Y. Lu, and C. K. Sun, Opt. Lett. 31, 308 (2006).

4. G. Brambilla, V. Finazzi, and D. Richardson, Opt. Express 12, 2258 (2004).

5. M. Sumetsky, Opt. Lett. 31, 870 (2006).

6. D. H. Auston, K. P. Cheung, and P. R. Smith, Appl. Phys. Lett. 45, 284 (1984).

7. D. H. Martin and E. Puplett, Infrared Phys. 10, 105 (1969). 